\title{
EFECTO DE APLICACIONES FOLIARES DE METANOL SOBRE EL RENDIMIENTO DE LECHUGA (Lactuca sativa L.)
}

\section{EFFECTS OF METHANOL FOLIAR APPLICATIONS ON THE GROWTH AND YIELD OF LETTUCE (Lactuca sativa L.)}

\author{
Mónica Solari ${ }^{1}$, Alfredo Rodríguez-Delfín, Sven E. Jacobsen, Hugo Soplín, Juan Guerrero y Salomón Helfgott.
}

\begin{abstract}
Resumen
Anteriores investigaciones, sugieren una incorporación por las plantas del carbono proveniente del metanol; por ello se diseñaron experimentos para determinar si su aplicación foliar afectaría el rendimiento y crecimiento en un cultivo hidropónico de lechuga (Lactuca sativa L.). Se utilizó metanol a los porcentajes de 10, 20,30,40, 50, 60, 70 y 80, con un testigo sin tratamiento. Se midieron el peso fresco, longitud del tallo floral, apertura estomatal y se anotaron otras observaciones correspondientes. Hubo variaciones estacionales, probablemente debido a las diferentes tasas de crecimiento vegetativo y de almacenamiento a lo largo del año, originadas por cambios de temperatura y luminosidad. A la cosecha, se registraron incrementos de peso radicular con las aplicaciones foliares de metanol, pero no incrementos de peso foliar. También se pudo observar aumento en la elongación del tallo floral en los tratamientos con máximas aplicaciones de metanol. No se pudo comprobar efectos sobre la apertura estomatal.
\end{abstract}

Palabras clave: metanol, aplicación foliar, lechuga, raíces, porómetro, respuesta dosis específica.

\begin{abstract}
Results obtained in previous studies suggested foliar incorporation of carbon coming from methanol. The objective of the present study was to determine the effects of foliar methanol application, on the growth and yield of an hydroponic culture of lettuce (Lactuca sativa L.). Methanol was used at percentages of 10, 20, 30, 40, 50, 60, 70 and 80. A control was also employed. Fresh weight, length of floral stem, and stomatal conductance were measured, and other pertinent observations were also registered. Seasonal changes, probably due to different growth rates and storage, influenced by variations in temperature and luminosity along the year, were also observed. These experiments revealed increments in root weight, but no foliar level weight increments were registered. Increments in the length of the floral stem related to methanol applications were also annotated. Effects on stomatal conductance were not conclusive.
\end{abstract}

Key words: methanol, foliar applications, lettuce, roots, porometer, dose specific response.

\section{Introducción}

El ciclo $\mathrm{C} 3$, es la ruta metabólica que emplean muchas especies de plantas en la fotosíntesis para captar el carbono ambiental en forma de dióxido de carbono, $\mathrm{CO}_{2}$, y transformarlo en carbohidratos. El $\mathrm{CO}_{2}$ es aceptado por la Ribulosa bifosfato (RuBP) que a través de una reacción mediada por la enzima rubisco genera dos moléculas de 3-fosfoglicerato (3PGA), las cuales, luego se reducen y producen hexosas base para la formación de carbohidratos que luego dan inicio a la regeneración del aceptor RuPB.

Sin embargo, la rubisco también se comporta como una oxigenasa en condiciones de baja concentración de $\mathrm{CO}_{2}$ y alta concentración de $\mathrm{O}_{2}$ y cataliza la primera fase del ciclo fotorrespiratorio. La fotorrespiración o ruta $\mathrm{C}_{2}$, es un proceso que ocurre en todas las plantas, principalmente en plantas $\mathrm{C}_{3}, \mathrm{y}$ se incrementa en condiciones de alta iluminación, altas temperaturas y bajo niveles de $\mathrm{CO}_{2}$. Básicamente es un proceso opuesto a la fotosíntesis.

Durante la fotorrespiración, la RuBP se oxida, produciendo una molécula de 3-PGA y otra de 2 Fosfoglicolato (2-PGA) en lugar de dos moléculas de
3-PGA. Para regenerar 3-PGA, ocurre una serie de reacciones a nivel celular entre los cloroplastos, peroxisomas y mitocondrias de las células fotosintéticas, reingresando así al ciclo $\mathrm{C}_{3}$ (Mathews \& van Holde, 1991). La liberación de $\mathrm{CO}_{2}$ ocurre al formarse una molécula de serina por cada dos moléculas de glicina en la mitocondria.

Para los científicos es un reto controlar la fotorrespiración de las plantas $\mathrm{C}_{3}$, la cual podría lograrse empleando ciertas prácticas agrícolas $\mathrm{o}$ mediante el empleo de la ingeniería genética. Dentro de las prácticas agrícolas, una posibilidad sería aumentar los niveles de $\mathrm{CO}_{2}$, alrededor de las hojas de las plantas $\mathrm{C}_{3}$, incrementando el nivel de $\mathrm{CO}_{2}$ ambiental. Esto puede bien suceder, se estima que, a finales del siglo, ocurra un doblamiento en la concentración de $\mathrm{CO}_{2}$ a nivel mundial (Watson et al., 1990).

El estudio de la ruta metabólica del carbono en la fotosíntesis (Benson, 1951; Bassham et al., 1954; Quayle et al., 1954), revelaron un rápido metabolismo del ${ }^{14} \mathrm{C}$ del metanol. Estudios comparativos de las tasas de fijación relativas del ${ }^{14} \mathrm{C}$ 
del $\mathrm{CO}_{2}$, y el ${ }^{14} \mathrm{C}$ del metanol en algas verdes unicelulares de Chlorella y Scenedesmus, concluyeron que el metanol es utilizado tan rápidamente como el $\mathrm{CO}_{2}$, para la producción de azúcares y aminoácidos (Calvin \& Benson, 1949). En estos experimentos iniciales, no se pudo demostrar con claridad, si las tasas fueron comparables, ó si la ruta metabólica para la conversión del metanol a sacarosa era la misma; sin embargo, experimentos subsiguientes en rebanadas de tejido de betarraga, hoja de betarraga, zanahoria, endospermo de soya y frijol (Cossins, 1964), revelaron que las plantas metabolizan rápidamente el metanol.

En investigaciones hechas con bacterias se concluyó que el metanol es oxidado a formaldehído y convertido a fructosa 6-fosfato (Cooney \& Levine, 1972).

La asimilación del metanol por microorganismos metilotrópicos es de forma rápida, donde el metanol es rápidamente oxidado a $\mathrm{CO}_{2}$, evitando la toxicidad por formaldehído. Las plantas también tienen la capacidad de la oxidación linear del metanol a $\mathrm{CO}_{2}$ (Cossins, 1980) que es rápidamente utilizado en la fotosíntesis, y también de la asimilación cíclica del metanol, donde interviene la transferencia de una especie fórmica activa, que integrada con el tetrahidrofolato, produce la glicina y homocisteina, que a su vez produce serina $y$ metionina respectivamente, el proceso continua luego su curso de transformación a glicerato y subsecuentemente a carbohidratos.

Nonomura \& Benson (1992), reportaron haber obtenido incrementos de rendimiento en diferentes cultivos tratados con metanol y sometidos a condiciones favorables para la fotorrespiración. Estos incrementos se dieron en cultivos $\mathrm{C}_{3}$, mas no en los cultivos $\mathrm{C}_{4}$. Por ello, el metanol empezó a ser investigado como un posible tratamiento para incrementar los niveles de $\mathrm{CO}_{2}$ alrededor de las hojas de plantas $\mathrm{C}_{3}$ y de esta manera reducir sus tasas de fotorrespiración.

Sin embargo, los resultados obtenidos por dichos autores no son concluyentes y en la actualidad existe mucha controversia respecto a la efectividad del metanol para bloquear o reducir la fotorrespiración y, por lo tanto, para aumentar los rendimientos en plantas $\mathrm{C}_{3}$ como algodón (Van Iersel et al., 1995), limón, pasto, lechuga, maíz, zanahoria, trigo, arveja y rabanito (Albrecht et al., 1995; Mc Giffen et al., 1995). Se ha informado, inclusive, una reducción en el rendimiento de la lechuga romana a dosis de $10 \mathrm{y}$ 30\% de metanol (McGiffen et al., 1995). McGiffen \& Manthey (1996), no pudieron corroborar los resultados de Nonomura \& Benson (1992) y sugirieron que los resultados obtenidos por dichos investigadores pueden haber sido producto de otros factores incluidos en los foliares, más no efectivamente del metanol.
Ante estos diversos resultados se decide investigar los efectos de aplicaciones foliares de metanol en el cultivo hidropónico de lechugas, para comprobar sus posibles efectos en el rendimiento y desarrollo de la planta.

\section{Material y métodos}

Los experimentos se llevaron a cabo en el Centro de Investigación de Hidroponía de la Universidad Nacional Agraria de la Molina, Lima, Perú, en condiciones de verano e invierno, durante los años 2000-2002.

Las plantas de lechuga fueron cultivadas a través de un sistema hidropónico denominado raíz flotante. Se usaron contenedores de madera de $0.4 \mathrm{~m} \mathrm{x} 0.4 \mathrm{~m} \mathrm{x}$ $0.1 \mathrm{~m}$, cubiertas en su interior con polietileno negro de 6 micras de espesor. Los contenedores fueron cubiertos con planchas de tecnopor de 1" de grosor. Cada plancha tenía cinco agujeros de $2.5 \mathrm{~cm}$ de diámetro.

Se sembraron semillas de lechuga de la variedad Dark Green Boston, en arena de cuarzo de 0.5-1.00 $\mathrm{mm}$ de diámetro, previamente lavada. A los 15 días las plántulas fueron transplantadas a un contenedor con agujeros de $1.50 \mathrm{~cm}$ de diámetro, con una densidad de 72 plántulas por contendor. Luego de 15 días, las plántulas fueron transplantadas a los contenedores definitivos, donde permanecieron alrededor de un mes.

La solución nutritiva se obtuvo a partir de la solución hidropónica La Molina (Rodríguez-Delfín et al., 2001), cuya concentración fue de: 190 ppm N; 35 ppm P; 200 ppm K; 150 ppm Ca; 45 ppm Mg; 70 ppm S; 1 ppm Fe; 0.5 ppm Mn; 0.5 ppm B; 0.15 ppm Zn; 0.1 ppm Cu; 0.05 ppm Mo.

La solución nutritiva se preparó con agua de pozo, la cual tuvo las siguientes características: $\mathrm{pH} 7.4$; CE $0.73 \mathrm{dS} / \mathrm{m} ; \mathrm{Cl}^{-} 3.5 \mathrm{me} / \mathrm{L} ; \mathrm{Ca}^{2+} 5.08 \mathrm{me} / \mathrm{L} ; \mathrm{Na}^{+} 0.73$ $\mathrm{me} / \mathrm{L} ; \mathrm{SO}_{4}=0.6 \mathrm{me} / \mathrm{L} ; \mathrm{Mg}^{2+} 1.04 \mathrm{me} / \mathrm{L} ; \mathrm{HCO}_{3}{ }^{-} 3.6$ me/L. (Rodríguez-Delfín et al., 2001). La solución nutritiva fue aireada con una bomba de aire de pecera.

Se efectuaron monitoreos del $\mathrm{pH}$, conductividad eléctrica (CE) y temperatura de la solución nutritiva, También se midió la resistencia estomatal con un porómetro LCA-2 System.

Para las aplicaciones se usó metanol de marca Merck $\left(\mathrm{CH}_{3} \mathrm{OH} ; 1 \mathrm{~L}=0.79 \mathrm{~kg} ; \mathrm{M}=31.04 \mathrm{~g} / \mathrm{mol}\right)$. Según el proyecto del experimento, se utilizaron siete dosis de metanol por vía foliar $(0,10,20,30,40,50$, 60 , y $80 \%$ ) más un testigo. Cada aplicación consistió de 10 aspersiones; el volumen por planta y por aplicación fue de $1.0 \mathrm{~mL}$

En todos los experimentos se empleó el Diseño Completo al Azar con 5 repeticiones (plantas) por tratamiento. Se realizaron los ANVA pertinentes y la prueba de Duncan $(\mathrm{P}<0.05)$ para determinar las diferencias entre promedios de tratamientos, para cada variable. 


\section{Resultados}

\section{Experimento Junio 2000}

Aunque se esperaba que el daño foliar aumentara conforme se incrementaba el porcentaje de metanol, esto no se hizo evidente aún con aplicaciones de $80 \%$ de metanol, pero sí se evidenció estrés fisiológico, obteniéndose $14 \%$ menos de peso foliar con respecto al testigo con los tratamientos $60 \%$ y $80 \%$, versus $4 \%$ y $9 \%$ menos con los tratamientos $20 \%$ y $40 \%$ respectivamente. Por otro lado, con $80 \%$, la CE de la solución nutritiva no bajó sustantivamente, quedando ésta en $2.1 \mathrm{dS} / \mathrm{m}$, sugiriendo una menor absorción de nutrientes; los tratamientos $0 \%$, $20 \%, 40 \%$ y $60 \%$ tuvieron una CE de $1.5 ; 1.5 ; 1.5$ y $1.6 \mathrm{dS} / \mathrm{m}$ respectivamente.

Tabla 1. Experimento Junio 2000. Efecto de las aplicaciones foliares de metanol en el peso fresco (gramos) de hojas, raíces y total obtenidos de las plantas de lechuga.

\begin{tabular}{cccccc}
\hline $\begin{array}{c}\text { Peso } \\
\text { fresco }\end{array}$ & $\begin{array}{c}\text { Metanol } \\
0 \%\end{array}$ & $\begin{array}{c}\text { Metanol } \\
20 \%\end{array}$ & $\begin{array}{c}\text { Metanol } \\
40 \%\end{array}$ & $\begin{array}{c}\text { Metanol } \\
60 \%\end{array}$ & $\begin{array}{c}\text { Metanol } \\
80 \%\end{array}$ \\
\hline $\begin{array}{c}\text { Raíces } \\
(\mathrm{g})\end{array}$ & $12.1 \mathrm{c}$ & $13.7 \mathrm{abc}$ & $17.0 \mathrm{ab}$ & $17.5 \mathrm{a}$ & $\begin{array}{c}14.8 \\
\mathrm{abc}\end{array}$ \\
$\begin{array}{c}\text { Foliar } \\
(\mathrm{g})\end{array}$ & $100.6 \mathrm{a}$ & $96.7 \mathrm{a}$ & $92.0 \mathrm{a}$ & $86.2 \mathrm{a}$ & $87.0 \mathrm{a}$ \\
$\begin{array}{c}\text { Total } \\
(\mathrm{g})\end{array}$ & $115.5 \mathrm{a}$ & $113.3 \mathrm{a}$ & $111.7 \mathrm{a}$ & $106.2 \mathrm{a}$ & $104.4 \mathrm{a}$ \\
\hline
\end{tabular}

Promedios con resultados significativos por la prueba de Duncan al $P=0.05$.

\section{Experimento Enero-2001}

El daño foliar se hizo evidente a partir de $60 \%$ de metanol en la aplicación foliar, se observó un decrecimiento inicial con respecto al testigo en el rendimiento total. No se encontraron diferencias significativas entre el testigo y los tratamientos de $40 \%, 50 \%$ y $60 \%$, pero si se encontraron diferencias entre tratamientos. En los tratamientos 0, 20, 40, 50, 60 y $80 \%$, la CE inicial fue de $2,1 \mathrm{dS} / \mathrm{m}$ y la CE final fue de 1,3 , a excepción del tratamiento $0 \%(1.4 \mathrm{dS} / \mathrm{m})$.

\section{Experimento Febrero 2002}

Resistencia estomática: Se midió la resistencia estomática $(\mathrm{s} / \mathrm{cm})$ de las plantas sometidas a diferentes dosis de metanol, con el propósito de evaluar la variabilidad del ingreso de $\mathrm{CO}_{2}$ vía foliar entre tratamientos. Los resultados se muestran en la Tabla 3.
Tabla 3. Experimento Febrero 2002. Efecto de las aplicaciones foliares del metanol sobre la resistencia estomatal $(\mathrm{s} / \mathrm{cm})$ en plantas de lechuga.

\begin{tabular}{|c|c|c|c|c|c|}
\hline Día & $0 \%$ & $20 \%$ & $40 \%$ & $50 \%$ & $80 \%$ \\
\hline 7 Feb. & $\begin{array}{c}0.66 \\
b\end{array}$ & $0.7 b$ & $1.31 \mathrm{ab}$ & $2.13 \mathrm{a}$ & $0.94 b$ \\
\hline 14 Feb. & $\begin{array}{c}0.72 \\
\mathrm{~b}\end{array}$ & $1.49^{\mathrm{a}}$ & $1.54 \mathrm{a}$ & $1.44 \mathrm{a}$ & $1.04 \mathrm{ab}$ \\
\hline $21 \mathrm{Feb}$ & $0.81 \mathrm{c}$ & $1.13 b c$ & $1.31 \mathrm{~b}$ & $2.01 \mathrm{a}$ & $1.29 \mathrm{bc}$ \\
\hline $\begin{array}{l}\text { Promec } \\
\text { resulta } \\
\text { prueba }\end{array}$ & \multicolumn{5}{|c|}{$\begin{array}{l}\text { con diferentes letras son diferentes } \\
\text { estadisticamente significativos por la } \\
\text { incan al } P=0.05 \text {. }\end{array}$} \\
\hline
\end{tabular}

Se diferenciaron los tratamientos de $40 \%$ y $50 \%$ metanol con respecto al testigo, comprobándose una mayor resistencia al libre pase de gases en las plantas tratadas con $40 \%$ y $50 \%$ metanol.

Tabla 4. Experimento Abril 2002. Resistencia (s/cm) estomática obtenida en plantas de lechuga sometidas a diferentes dosis de metanol.

\begin{tabular}{ccccc}
\hline Día & $0 \%$ & $20 \%$ & $50 \%$ & $80 \%$ \\
\hline 8 Abril & $0.64^{\mathrm{a}}$ & $0.48 \mathrm{ab}$ & $0.35 \mathrm{~b}$ & $0.43 \mathrm{~b}$ \\
12 Abril & $0.53 \mathrm{a}$ & $0.41 \mathrm{ab}$ & $0.21 \mathrm{c}$ & $0.28 \mathrm{bc}$ \\
\hline
\end{tabular}

Promedios con diferentes letras son diferentes resultados porla prueba de Duncan al $P=0.05$.

\section{Experimento Abril 2002:}

También se midió la resistencia estomática (Tabla 4), y los resultados de la elongación del tallo floral (Tabla 5).

Resistencia estomática: Los resultados de los experimentos de Febrero y Abril del 2002, muestran diferencias estadísticas entre tratamientos, mas los resultados no se duplicaron.

Tallo floral: Se diferenciaron significativamente los tratamientos de $50 \%$ y $80 \%$ metanol con respecto al testigo, demostrando una mayor elongación de tallo en los tratamientos con metanol.

Recapitulando, podemos decir:

- En el experimento de invierno (Junio, 2000), se pudo demostrar un incremento de peso radicular con el tratamiento $60 \%$ metanol, comparado con el 
testigo $(0 \%)$, con tratamientos menores $(20 \%)$ y mayores $(80 \%)$. Respuesta curva tipo "loma".

- En los resultados del experimento de verano (Enero, 2001) con la dosis de 50\% metanol se pudo demostrar un incremento de peso total comparado con otros tratamientos de $20 \%$ y $80 \%$. Se trata también de una respuesta curva tipo "loma".

- En estos dos experimentos, el metanol habría afectado negativamente el rendimiento de la planta aún en bajas concentraciones, y habría favorecido a dosis específicas para este cultivo $(40 \%, 50 \%$, $60 \%$ ), el rendimiento de la planta, superando incluso, el efecto tóxico del mismo. Pero en ningún caso llegó a superar al testigo.

- En los experimentos hechos bajo media luminosidad (Junio 2000 y Enero 2001), se pudo observar que la dosis de $50 \%$ metanol provoca un aumento significativo en el peso radicular de la planta.

- El hecho que el metanol haya incrementado el peso radicular, puede relacionarse con el trabajo de Ziska et al. (1997), quienes experimentaron diferentes niveles de $\mathrm{CO}_{2}$ ambiental en el cultivo de arroz y obtuvieron un incremento en el peso radicular, relacionado con mayores concentraciones de $\mathrm{CO}_{2}$. Según Rogers et al. (1992), este incremento se debe a que las raíces son reservorios de exceso de carbono incorporado en condiciones elevadas de $\mathrm{CO}_{2}$.

- En el experimento de Junio del 2000, se observó una menor disminución en la $\mathrm{CE}$ en invierno comparado con lo obtenido en verano. Estos resultados están de acuerdo con las explicaciones Kennedy \& Gonsalves (1988), que una restringida absorción de iones a bajas temperaturas es principalmente debida a una baja movilidad en la fluidez de la membrana plasmática y una baja actividad en la bomba de protones de la misma.

- Las observaciones hechas al peso radicular y el comportamiento de la $\mathrm{CE}$ del experimento de Junio de 2000, demostraría que el crecimiento radicular y el transporte iónico no están directamente relacionados $0 \%$ vs. $40-50 \%$ (Bravo \& Uribe, 1981; Engels \& Marschner, 1992).

- Cuando se probaron varias aplicaciones de metanol, no se pudo comprobar que un mayor número de aplicaciones, favorece o no al incremento de peso fresco.

- No se pudo comprobar una mayor conductancia estomática en la planta con la aplicación del metanol (Experimentos de Febrero y Abril de 2002).

- Se pudo observar un incremento en la elongación del tallo floral de las plantas con aplicaciones de metanol versus el testigo (Experimento de Abril de 2002).
- A concentraciones de $80 \%$ ó más, el efecto tóxico del metanol se hace evidente por la caída en el rendimiento de las plantas $\mathrm{y}$, en algunos casos, por malformación de hojas.

- Ningún tratamiento superó al testigo en peso total, corroborando los resultados de McGiffen et al. (1995), recordar el decrecimiento mencionado a bajos niveles $10 \%$ y $30 \%$.

Tabla 5. Experimento Abril 2002. Efectos de aplicaciones foliares de metanol sobre la longitud de tallo.

\begin{tabular}{ccccc}
\hline Tratam. & $0 \%$ & $20 \%$ & $50 \%$ & $80 \%$ \\
\hline $\mathrm{cm}$ & $38.5 \mathrm{~b}$ & $42.63 \mathrm{ab}$ & $48.88 \mathrm{a}$ & $50.0 \mathrm{a}$ \\
\hline Promedios & con diferentes letras & son diferentes \\
resultados significativos por la prueba de Duncan \\
al $P=0.05$.
\end{tabular}

\section{Discusión}

¿Qué significarían estos resultados? incremento radicular mas no incremento total, mayor elongación del tallo, e incoherencia en resultados estomatales.

La aplicación promedio de metanol en los experimentos fue de $3 \times 1 \mathrm{ml}$ de metanol acuoso cada una, haciendo un total de $3 \mathrm{ml}$ de metanol acuoso por planta. Para hacer un cálculo referencial, en una dosis de $50 \% \mathrm{v} / \mathrm{v}$, la cantidad de metanol en los $3 \mathrm{ml}$ es de $790 \mathrm{mg}$ de metanol/ ml "Metanol-Merck" *0.50 $(50 \% \mathrm{v} / \mathrm{v})=395 \mathrm{mg}$ metanol puro $/ \mathrm{mL} * 3$ aplicaciones $=1.185 \mathrm{~g}$ de Metanol puro $=0.488 \mathrm{~g}$ de carbono total aplicado/planta.

Si la relación entre peso fresco y peso seco para la lechuga es de $5.2 \%$ y, la relación de carbono con los otros componentes de la planta es de $43.6 \%$ entonces, $0.488 \mathrm{~g}$ de carbono aplicado (asumiendo una incorporación total se traduciría en 0.488 g x 2.29 (de carbono a compuestos de la planta) $* 19.23$ (de peso seco a fresco) $=21.52 \mathrm{~g}$, el cual sería el incremento máximo en peso fresco. En un peso promedio de 200 g por planta de lechuga, este incremento se traduciría en un $10 \%$ de incremento, lo cual no se observó.

$\mathrm{Si}$ el efecto hubiera sido el de más carbono absorbido (traduciéndose en un aumento de peso), se podría sugerir que la fotorrespiración hubiera sido controlada con las aplicaciones de metanol; hipótesis que ha sido formulada por Nonomura \& Benson (1992), quienes señalaron la 'no-respuesta' de plantas $\mathrm{C}_{4}$ a los tratamientos.

No se pudo comprobar que el metanol induzca una mayor apertura estomatal $\mathrm{y}$, de esta manera permitir una mayor cantidad de carbono absorbido, ya que las lecturas del porómetro dieron dos respuestas opuestas (en un caso sí, en otro no).

La asimilación del carbono proveniente del metanol habría sido por la misma planta ó ayudada por la bacteria metilotrópica Methylobacterium (Holland \& Polacco, 1994), la cual facilitaría la conversión de metanol en formato y luego en $\mathrm{CO}_{2}$, 
forma asimilable para las hojas de las plantas. Según Taiz \& Zeiger (1998), estas bacterias se encuentran presentes en diferentes formas en la superficie de las hojas y los espacios intercelulares de las plantas.

Quayle (1980), menciona también un metabolismo de metanol por las bacterias, el cual interviene una especie fórmica activa que junto con un tetrahidrofolato transforma la glicina a serina $\mathrm{y}$ continúa luego su curso de transformación a glicerato, PGA-PEP y combinándose finalmente con $\mathrm{CO}_{2}$ a compuestos $\mathrm{C}_{4}$

También existe la posibilidad de que la presencia de estas bacterias activadas por el metanol hayan sido inducidas a producir citokininas, las cuales podrían explicar el incremento en la masa foliar de previos experimentos, y explicaría la falta de correlación que tienen unos autores sobre la cantidad de metanol aplicado con los incrementos de biomasa reportados.

En este trabajo, no se ha registrado un incremento foliar en los experimentos pero sí, en algunos casos, un incremento en la masa radicular. Por ello, se hace otro planteamiento donde se examina la posibilidad de una respuesta auxínica como consecuencia de las aplicaciones de metanol.

Las respuestas fisiológicas observadas fueron de respuestas tipo "loma" en los pesos radiculares de los diferentes tratamientos de metanol, excepto el testigo (Experimentos de Junio de 2000 y Enero de 2001) y de elongación de tallo (Experimento de Abril de 2002), las cuales simulan un comportamiento hormonal similar al de la auxina natural AIA (ácido 3indolacético). La elongación de tallo podría también indicar un comportamiento giberélico, o simplemente una respuesta a las mayores reservas radiculares de las plantas tratadas con metanol, pero se necesita tener mayores evidencias de estas hipótesis probados en otros niveles.

Las auxinas son sintetizadas en los terminales apicales de los tallos en crecimiento. Entre otras funciones, las auxinas estimulan el crecimiento del tallo principal inhibiendo el desarrollo de tallos laterales, las auxinas afectan el proceso usual de formación de raíces que ayudan a balancear el crecimiento de la raíz y el sistema aéreo. Además, existe buena evidencia de que las auxinas procedentes del tallo influyen mucho en la iniciación de la raíz.

Indicios de estos efectos de mayor crecimiento del tallo principal se observaron en el experimento de Abril de 2002, en el que tratamientos con aplicaciones de metanol exhibieron un mayor largo de tallo, y el indicio de mayor desarrollo radical pudo ser observado en los Experimentos de Junio 2000 y Enero de 2001.

\section{Conclusiones}

Se concluye que el metanol afecta el crecimiento y desarrollo de la lechuga, y la respuesta tiene relación con la dosis aplicada. Aunque con las aplicaciones foliares de metanol no se ha incrementado el peso fresco de las hojas, pero sí el peso fresco de las raíces, y una respuesta dosis específica en los pesos total y foliar, y comportamiento del tallo floral.

El cómo afecta este crecimiento es más elusivo. Las posibilidades planteadas han sido:

1. Contrarresta la fotorrespiración

2. Ingreso facilitado del $\mathrm{CO}_{2}$ por las metilbacterias

3. Respuesta de crecimiento debido a citokininas

4. Respuesta de crecimiento en algunos casos semejante al de una respuesta hormonal (posiblemente auxínica o giberélica), hipótesis sugerida por los resultados del presente trabajo.

Al no haber visto cambios en el peso total, y más bien haber observado cambios en la distribución del peso, los resultados de este ensayo concluyen en que el metanol promueve un comportamiento que se asemejaría más bien a una respuesta de tipo hormonal, y que se recomienda más a fondo investigar los efectos del metanol ambiental o sus derivados en las plantas y cultivos agrícolas.

\section{Agradecimientos}

Al Dr. Pedro Aguilar por sus valiosas sugerencias en la revisión del manusctrito, y al Ingeniero Luis Pizarro por el apoyo técnico necesario para su presentación.

\section{Literatura citada}

Albrecht S.L.C.L., Douglas J.R., Llepper E.L., Rasmussen P.E., Rickmant R.W., Smiley R.W., Wilkins D.E. \& Wysocki D.J. 1995. Effect of Foliar Methanol Applications on Crop Yield. Crop Sci. 35: 1642-1646.

Bassham J.A, Benson A,A, Kay L.D, Harris A.Z, Wilson A.T.\& Calvin M. 1954. The Path of Carbon in Photosynthesis. XX1. The Cyclic Regeneration of Carbon Dioxide Acceptor. J.Am. Chem. Soc. 76: 1760-1770.

Benson A.A. 1951. Identification of Ribulose in $\mathrm{C}^{14} \mathrm{O}_{2}$ Photosynthesis Products. J. Am. Chem. Soc. 73: 29-71.

Bravo F.P. \& Uribe E.G. 1981. Temperature dependence of the concentration kinetics of absorption of phosphate and potassium in corn roots. Plant Physiol. 67: 815-819.

Calvin M. \& Benson A.A. 1949. The Path of Carbon in Photosynthesis IV: The Identity and Sequence of Intermediates in Sucrose Synthesis. Science.109: 140-142.

Cooney C.L. \& Levine D.W. 1972. Microbial utilization of methanol. Adv. Appl. Microbiol. 15: 337-365.

Cossins E.A. 1964. The utilization of carbon-1 compound by plants. The metabolism of C-14 and its role in amino acid biosynthesis. Canadian Journal of Biochemistry. 42: 1793-1802.

1980. One carbon metabolism. In: DD.Davies (ed.) The Biochemistry of Plants. Vol2. Academic. NY. : 364-418. 
Engels C. \& Marschner H. 1992. Adaptation of potassium translocation into the shoot of maize Zea mays to shoot demand: evidence for xylem loading as a regulating step. Physiol. Plant 86: 263-268.

Holland M.A. \& Polacco J.C. 1994. PPFMs and other covert contaminants: is there more to plant physiology than just plant?. Annual Rev. Plant Phys. Plant Mol. Biol. 45: 197-209.

Kennedy C.D. \& Gonsalves F.A.N. 1988. $\mathrm{H}^{+}$efflux and trans-root potential measured while increasing the temperature of solutions bathing excised roots of Zea mays. J.Exp.Bot. 39: 37-49.

Mathews C. \& van Holde K.E. 1991. Biochemistry. The Benjamin/Cummings Publishing Company.

McGiffen M., Manthey J., Faber B., Downer J. Sakowich N. \& Aguiar J. 1995. Field tests of methanol as a crop yield enhancer. Hort Science. 30(6): 1225-1228.

McGiffen M. \& Manthey J. 1996. The role of methanol in promoting plant growth: A current evaluation. HortScience. 31(7): 1092-1096.

Nonomura A.M. \& Benson A.A. 1992. The path of carbon in photosynthesis: Improved crop yields with methanol. Proc. Natl. Acad. Sci. USA. 89: 9794-98.

Quayle J.R, Fuller R.C.,Benson A.A., \& Calvin M. 1954. Enzymatic carboxylation of ribulose diphosphate. J. Am. Chem. Soc. 76: 3610-3611.
Quayle J.R. 1980. Microbial assimilation of C1 compound. Biochem. Soc. Trans. 8: 1-10.

Rodríguez-Delfín A., Hoyos M. \& Chang M. 2001. Soluciones Nutritivas en Hidroponía: Formulación y Preparación. CIHNM, UNALM. Lima, Perú.

Rogers H.H., Peterson C.M.,Mc Crimmon J.N., \& Curo J.D. 1992. Response of plant roots to elevated atmospheric carbon dioxide. Plant Cell Environment. 15: 749-752.

Taiz L. \& Zeiger E. 1998. Are Symbiotic Bacteria the Source of Plant Hormonal Cytokinins?. Plant Physiology.

Van Iersel M., Heitholt J., Wells R. \& Oosterhuis D.1995. Foliar methanol applications to cotton in the southeastern United States: leaf physiology, growth, and yield components'. Agron. J. 87: 1157-1160.

Watson R.T., Rodhe H., Oescheger H. \& Siegenthaler U. 1990. Greenhouse gases and aerosols. In J.T. Houghton et al (ed.) Climate change: The IPCC scientific assessment. Cambridge Univ. Press, Cambridge. : 1-40.

Ziska L.H., Namuco O., Moya T. \& Quilang J. 1997. Growth and yield response of field-grown tropical rice to increasing carbon dioxide and air temperature. Agron. J. 89: 45-53.

\footnotetext{
${ }^{1}$ Centro de investigación de Hidroponía y Nutrición Mineral. Universidad Nacional Agraria La Molina, Lima. Mg. Sc. Mónica Solari (511) 441-8621/ 9876-1948/453-5053 (of). Correo electrónico. msolari@imarpe.gob.pe / solarimonica@yahoo.com
} 